\section{(6) OPEN ACCESS}

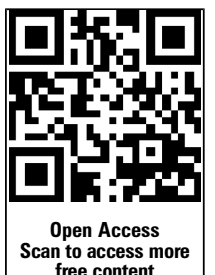

Handling editor Tore K Kvien

- Additional material is published online only. To view please visit the journal online (http://dx.doi.org/10.1136/ annrheumdis-2013-203600).

1 Department of Biochemistry, University of Otago, Dunedin, New Zealand

${ }^{2}$ Department of Rheumatology, Middlemore Hospital, Auckland, New Zealand ${ }^{3}$ Department of Medicine, University of Otago, Wellington, New Zealand ${ }^{4}$ Department of Medicine, University of Otago, Dunedin, New Zealand

${ }^{5}$ Department of Medicine, University of Auckland, Auckland, New Zealand ${ }^{6}$ Department of Medicine, University of Otago, Christchurch, New Zealand

\section{Correspondence to} Dr Tony R Merriman, Department of Biochemistry, University of Otago, Dunedin, 9054, New Zealand; tony.merriman@otago.ac.nz

CB and AJP-G contributed equally.

Received 11 March 2013 Revised 25 June 2013 Accepted 15 July 2013 Published Online First 11 September 2013

\title{
Sugar-sweetened beverage consumption: a risk factor for prevalent gout with SLC2A9 genotype- specific effects on serum urate and risk of gout
}

\author{
Caitlin Batt, ${ }^{1}$ Amanda J Phipps-Green, ${ }^{1}$ Michael A Black, ${ }^{1}$ Murray Cadzow, ${ }^{1}$ \\ Marilyn E Merriman, ${ }^{1}$ Ruth Topless, ${ }^{1}$ Peter Gow, ${ }^{2}$ Andrew Harrison, ${ }^{3}$ John Highton, ${ }^{4}$ \\ Peter Jones, ${ }^{5}$ Lisa Stamp, ${ }^{6}$ Nicola Dalbeth, ${ }^{5}$ Tony R Merriman ${ }^{1}$
}

\section{ABSTRACT}

Objective Consumption of high fructose corn syrup (HFCS)-sweetened beverages increases serum urate and risk of incident gout. Genetic variants in SLC2A9, that exchanges uric acid for glucose and fructose, associate with gout. We tested association between sugar (sucrose)-sweetened beverage (SSB) consumption and prevalent gout. We also tested the hypothesis that SLC2A9 genotype and SSB consumption interact to determine gout risk.

Methods Participants were 1634 New Zealand (NZ) European Caucasian, Māori and Pacific Island people and 7075 European Caucasians from the Atherosclerosis Risk in Communities (ARIC) study. NZ samples were genotyped for rs 11942223 and ARIC for rs6449173. Effect estimates were multivariate adjusted.

Results SSB consumption increased gout risk. The OR for four drinks/day relative to zero was 6.89 ( $p=0.045)$, $5.19(p=0.010)$ and $2.84(p=0.043)$ for European Caucasian, Māori and Pacific Islanders, respectively. With each extra daily SSB serving, carriage of the goutprotective allele of SLC2A9 associated with a $15 \%$ increase in risk $(p=0.078)$, compared with a $12 \%$ increase in non-carriers $(p=0.002)$. The interaction term was significant in pooled ( $p_{\text {Interaction }}=0.01$ ) but not meta-analysed ( $p_{\text {Interaction }}=0.99$ ) data. In ARIC, with each extra daily serving, a greater increase in serum urate protective allele carriers $\left(0.005\left(p=8.7 \times 10^{-5}\right)\right.$ compared with $0.002(p=0.016) \mathrm{mmol} / \mathrm{L})$ supported the gout data $\left(p_{\text {Interaction }}=0.062\right)$.

Conclusions Association of SSB consumption with prevalent gout supports reduction of SSB in management. The interaction data suggest that SLC2A9mediated renal uric acid excretion is physiologically influenced by intake of simple sugars derived from SSB, with SSB exposure negating the gout risk discrimination of SLC2A9.

\section{INTRODUCTION}

A central risk factor for gout is hyperuricaemia. ${ }^{1}$ The dominant mechanism of determining hyperuricaemia is by regulation of uric acid excretion, primarily renal, but also involving the gut. ${ }^{2}$ Regulation of serum urate is under genetic and environmental control, ${ }^{3}$ with 28 loci that influence serum urate levels identified as a result of genomewide association scanning in European Caucasian. ${ }^{4}$ The major genetic regulator is SLC2A9, which encodes the GLUT9 renal molecule that transports uric acid in exchange with glucose and fructose. ${ }^{5} 6$ Variants within SLC2A9 explain 3.70\% of the phenotypic variance in serum urate levels in European Caucasian and are strongly associated with gout in European Caucasian, NZ Māori and NZ Pacific Islander sample sets. ${ }^{4}$

There is positive association between higher consumption of beverages sweetened with fructosecontaining sweeteners, such as high fructose corn syrup (HFCS), and increased risk of incident gout in men and women. ${ }^{8-10}$ For exposure to $\geq 2$ sugar-sweetened beverages (SSB) per day, within the Health Professionals Follow-up Study there was a 1.78 -fold increased risk of gout in men, ${ }^{8}$ and in the Nurses' Health Study a 3.05-fold increased risk in women. ${ }^{9}$ The increased risk of gout resulting from exposure to SSB is mediated, at least in part, by an effect on serum urate levels in both men and women, ${ }^{10}$ and acute and hypercaloric ingestion of fructose raises serum urate levels. ${ }^{11}{ }^{12}$ Fructose is metabolised in the liver, initiated by the unregulated phosphorylation of fructose to fructose-1phosphate. The subsequent depletion of ATP and increase in AMP is proposed to lead to increased serum urate via the catabolism of AMP. ${ }^{913}{ }^{14}$ Acute ingestion of HFCS-sweetened soft drinks increases the renal fractional excretion of uric acid (FEUA), ${ }^{15}$ suggesting a response of renal transport to either uric acid or fructose. Given that SLC2A9 also transports fructose and glucose, ${ }^{5}{ }^{6}$ and that SLC2A9 genotype influences serum urate and FEUA response to a fructose load, ${ }^{16}$ it is possible that fructose and glucose can both directly interfere with SLC2A9-mediated renal uric acid excretion.

There were two aims of this study. One, to replicate association between consumption of SSB and prevalent gout in a country where soft drinks are sweetened with sucrose and not HFCS. The second aim was to test for non-additive interaction between SSB consumption and SLC2A9 genotype.

\section{SUBJECTS AND METHODS \\ Subjects}

Clinical and survey information was collected from 1634 individuals from 2006 to 2011 (table 1). Cases $(n=925)$ were recruited from rheumatology clinics, workplaces and community focal points from the Auckland, Bay of Plenty, Wellington, 
Table 1 Baseline demographic and clinical characteristics of study participants by ethnicity

\begin{tabular}{|c|c|c|c|c|c|c|c|c|}
\hline \multirow[b]{2}{*}{ Individuals (n) } & \multicolumn{2}{|c|}{ NZ European Caucasian } & \multicolumn{2}{|c|}{ NZ Māori } & \multicolumn{2}{|c|}{ NZ Pacific Islander } & \multicolumn{2}{|c|}{ ARIC European Caucasian } \\
\hline & Obs & 592 & Obs & 502 & Obs & 540 & Obs & 7075 \\
\hline Cases & & 412 & & 190 & & 323 & & 148 \\
\hline Controls & - & 180 & - & 312 & - & 217 & - & 6927 \\
\hline Age mean, years (range) & & $57.9(17-94)$ & & $47.1(17-81)$ & & $44.6(17-86)$ & & $53.8(44-65)$ \\
\hline Cases & 592 & $63.8(23-94)$ & 501 & $54.2(23-81)$ & 539 & $47.7(18-81)$ & 7075 & $54.5(45-65)$ \\
\hline Controls & & $44.4(17-79)$ & & $42.7(17-80)$ & & $39.9(17-86)$ & & $53.8(44-65)$ \\
\hline Sex (\% male) & & 68.3 & & 51.0 & & 75.9 & & 47.4 \\
\hline Cases & 590 & 77.8 & 498 & 80.3 & 539 & 87.9 & 7075 & 75.0 \\
\hline Controls & & 46.7 & & 33.2 & & 57.9 & & 46.8 \\
\hline BMI mean, (range) & & $29.4(19.2-62.7)$ & & $33.2(18.1-77.0)$ & & $36.4(19.4-71.1)$ & & $26.4(14.4-54.6)$ \\
\hline Cases & 582 & $29.8(19.2-61.7)$ & 496 & $35.0(21.7-72.7)$ & 530 & $37.4(21.5-71.1)$ & 7073 & $28.0(21.1-40.5)$ \\
\hline Controls & & $28.3(19.5-62.7)$ & & $32.1(18.1-77.0)$ & & $34.9(19.4-65.1)$ & & $26.3(14.4-54.6)$ \\
\hline Kidney disease (\%) & & 12.8 & & 8.6 & & 8.5 & & 1.2 \\
\hline Cases & 579 & 17.6 & 486 & 18.5 & 518 & 12.9 & 6757 & 1.4 \\
\hline Controls & & 1.7 & & 2.7 & & 1.9 & & 1.2 \\
\hline High blood pressure (\%) & & 38.0 & & 32.4 & & 34.5 & & 12.7 \\
\hline Cases & 585 & 49.9 & 488 & 57.6 & 528 & & 7037 & 20.3 \\
\hline Controls & & 10.2 & & 17.1 & & 15.6 & & 12.6 \\
\hline
\end{tabular}

Christchurch and Dunedin areas of New Zealand (NZ), and had gout ascertained using the American Rheumatology Association Criteria. ${ }^{17}$ Controls with no self-reported history of gout $(n=709)$ were convenience sampled from workplaces and community focal points in the Auckland region of NZ. Ancestry was self-reported. The research was approved by the NZ Multi-Region Ethics Committee (MREC 05/10/130) and all subjects gave written informed consent.

Subjects were included from the Atherosclerosis Risk in Communities (ARIC) study and comprised 7075 controls of European Caucasian ancestry who self-reported as having not taken blood pressure-lowering medication in the previous 2 weeks (at either of exam 1 (1987-1989) or 4 (1996-1998)). Primary gout cases $(n=148)$ were European Caucasian subjects who self-reported at Exam 4 as having physician-diagnosed gout and did not report as having taken blood pressure-lowering medication in the previous 2 weeks (at either of exam 1 or 4 ).

\section{Data collection and selection of exposure groups}

Collection of exposure, genetic, anthropomorphic and relevant clinical data is described in the online supplementary material.

\section{Data analysis}

Regression analysis was used to investigate relationships between the variables in this study. For continuous response variables, this involved standard linear regression; for binary response variables (ie, discrete variables with only two levels), logistic regression was used. $P_{\text {trend }}$ was the $\chi^{2} \mathrm{p}$ value associated with the SSB variable in regression analysis. Both main effects and interaction terms were included in the models where appropriate. Coefficients with $\mathrm{p}$ values $\leq 0.05$ were considered to indicate a significant association between the response and explanatory variables. For discrete explanatory variables with more than two levels, significance was achieved if at least one of the variable's model coefficients achieved a $p$ value of $\leq 0.05$. The adjusted OR for a single explanatory variable was obtained by using the logistic regression coefficient. An adjusted OR for the same variable was obtained by including additional variables in the logistic regression model, again taking the value of the model coefficient relating to the variable in question. In order to make ORs interpretable for multilevel discrete variables, these variables were converted to binary (two-level) form prior to analysis. Logistic regression analysis was used to assess the association between gout (binary response variable), and SSB consumption and SLC2A9 genotype (explanatory variables) and linear regression analysis was used to assess the association between serum urate levels (response variable), and SSB consumption and SLC2A9 genotype (explanatory variables). Unstandardised regression coefficients are reported. Main effects were included in the model for SSB consumption and SLC2A9, and for the covariates age, sex, BMI, kidney disease, high blood pressure, alcohol and fruit consumption. Individuals with missing data from any variable were excluded from analysis. An interaction term between SSB consumption (as a continuous variable) and SLC2A9 genotype (binary: carriage and non-carriage of the serum urate-lowering and gout-protective minor allele) was also included. Results from different datasets were combined by both pooling data and adjusting for the dataset in association analysis, and Mantel-Haenszel meta-analysis with random effects. STATA software V.8.0 was used for all statistical analysis (http://www.stata.com).

\section{RESULTS}

\section{SSB consumption and risk of gout}

Table 1 presents clinical and demographic characteristics of study subjects and online supplementary table S1 presents characteristics of the sample sets according to SSB consumption. The level of SSB intake was lower in the ARIC sample; two possible explanations, at least in part, for this are: one, that the ARIC exposure data were collected 1987-1989 and the NZ exposure data 2006-2011 and two, the ARIC sample set consists of individuals 44 years of age or older, whereas the NZ samples consisted of younger individuals (table 1), who consume more SSB (see online supplementary table S1).

Testing for association of SSB consumption with gout and unadjusted ORs provided no evidence that increasing intake of 
sucrose-sweetened and HFCS-sweetened beverages was associated with increasing risk of gout in any of the sample sets (table 2; $p_{\text {trend }}=0.56,0.17,0.16$ and 0.20 for the NZ European Caucasian, Māori, Pacific Islander and ARIC sample sets, respectively). Multivariate adjustment by gout risk factors BMI, age, sex, alcohol and fruit consumption, kidney disease and hypertension provided stronger evidence for association in all $\mathrm{NZ}$ ancestral groups (all $\mathrm{p}_{\text {trend }}<0.11$; OR for gout risk for 4 $\mathrm{SSB} /$ day relative to $0 \mathrm{SSB} /$ day in $\mathrm{NZ}$ European Caucasian, Māori and Pacific Islander sample sets were $6.89(\mathrm{p}=0.045)$, $5.19(\mathrm{p}=0.010)$ and $2.84(\mathrm{p}=0.043)$, respectively). In the ARIC cohort, the OR for $\geq 5 \mathrm{SSB} /$ day of $2.31 \quad(\mathrm{p}=0.20)$ was comparable with that for the NZ European Caucasian group $(\mathrm{OR}=2.38, \mathrm{p}=0.20)$. Adjustment by individual covariates (see online supplementary table S2) revealed age to be the major confounder-a combination of younger people consuming more SSBs (see online supplementary table S1: in controls the change in age per increase in SSB category were $\beta=-3.21$, $p=1.2 \times 10^{-4} ; \beta=-1.77, p=3.7 \times 10^{-5} ; \beta=-1.40, p=7 \times 10^{-3}$; $\beta=-0.50, p=3.6 \times 10^{-14}$ for NZ European Caucasian, Māori, Pacific Islander and ARIC, respectively), and NZ control sample sets having a younger age of recruitment than cases (table 1) would have obscured association between SSB and risk of gout in the unadjusted analysis.

\section{Testing for non-additive interaction between SSB consumption, SLC2A9 genotype and risk of gout}

The SLC2A9 gene was genotyped (see online supplementary table S3). Within the NZ Pacific Islanders, NZ European Caucasians and ARIC, and all datasets combined, there was a greater increase in risk of gout per increase in daily SSB consumption category in the presence of the gout protective and serum urate-lowering allele than in the group homozygous for the (major) gout risk and serum urate-increasing allele (table 3; $71 \%$ vs $11 \%$ in NZ Pacific Islander; $27 \%$ vs $16 \%$ in NZ European Caucasian; $16 \%$ vs 5\% in ARIC). In NZ Māori, there appeared to be an opposing effect, with the increase in risk of gout per unit increase in SSB consumption greater in the major allele homozygous group $(\mathrm{OR}=1.19)$ than the minor allele-carrying group $(\mathrm{OR}=0.76)$. Interaction analysis of SLC2A9 genotype (presence or absence of the protective (minor) allele $\mathrm{C}^{7}$; rs11942223 in NZ and rs6449173 in ARIC) and SSB as a continuous measure did not reveal consistent evidence for non-additive interaction according to the method of combining datasets (table 3 ; $\mathrm{p}_{\text {Interaction }}=0.010$ in pooled combined groups, $\mathrm{p}_{\text {Interaction }}=0.99$ by meta-analysis of individual interaction terms from the four sample sets). There was evidence for heterogeneity in interaction terms $\left(p_{\text {Het }}=0.025\right)$, with the Māori sample set driving the heterogeneity $\left(\mathrm{p}_{\text {Interaction }}=0.39\right.$, $\mathrm{p}_{\mathrm{Het}}=0.17$ excluding the Māori samples).

\section{Testing for non-additive interaction between SSB consumption, SLC2A9 genotype and serum urate}

Given evidence for a difference in effect according to genotype between SLC2A9 and SSB consumption with gout as outcome, we next investigated if a similar phenomenon occurred with serum urate as outcome. In the ARIC cohort (excluding people classified with gout), there was a positive correlation between SSB consumption and serum urate levels, with a $0.003 \mathrm{mmol} / \mathrm{L}$ $\left(p=6.9 \times 10^{-6}\right)$ increase in serum urate per change in SSB category (table 4). Each copy of the rs6449173 minor allele (C-a perfect surrogate $\left(r^{2}=1.0\right)$ for the $C$ allele of $r s 11942223$ in European Caucasian) decreased serum urate by $0.025 \mathrm{mmol} / \mathrm{L}$ $\left(\mathrm{p}<1 \times 10^{-8}\right)$. With increasing SSB consumption in the overall cohort the presence of the normally serum urate-lowering $\mathrm{C}$ allele conferred a higher increase in serum urate per change in SSB category $\left(0.005 \mathrm{mmol} / \mathrm{L}, \mathrm{p}=8.7 \times 10^{-5}\right)$ compared to those without the $\mathrm{C}$ allele $(0.002 \mathrm{mmol} / \mathrm{L}, \mathrm{p}=0.016)$. There was a trend towards significance for non-additive interaction between rs6449173 and SSB in determining serum urate levels $\left(\beta_{\text {Interaction }}=0.003, p=0.062\right)$. Artificially sweetened soft drinks did not raise serum urate levels $(\beta=0.000, p=0.48)$, nor was there any indication of a genotype-specific effect of artificially sweetened soft drinks on serum urate $(\beta=0.000, p=0.41$ in the group not carrying the $C$ allele; $\beta=-0.004, p=0.45$ in the $\mathrm{C}$ allele carrier group; $\beta_{\text {Interaction }}=-0.001, \mathrm{p}=0.39$ ).

\section{DISCUSSION}

We demonstrate association between sucrose-sweetened soft drink consumption and risk of gout in the Polynesian (Māori and Pacific Islanders) and European Caucasian ancestral groups of NZ, with risk independently conferred when age, gender, BMI, alcohol and fruit consumption, kidney disease and hypertension were taken into account. We also found an indication of non-additive interaction between SLC2A9 genotype and SSB consumption in determining the risk of gout when analysing genotype-specific groups. However, the statistical support for non-additive interaction in risk of gout was inconsistent (table $3 ; \mathrm{p}_{\text {Interaction }}=0.01$ in pooled individual-level data with adjustment by dataset and $\mathrm{p}_{\text {Interaction }}=0.99$ in meta-analysis of interaction terms from the four individual datasets). There was evidence for heterogeneity between the four sample sets meta-analyzed $\left(\mathrm{p}_{\mathrm{Het}}=0.025\right.$; the NZ Māori sample set driving the heterogeneity had a significant interaction term of $\mathrm{p}_{\text {Interaction }}=0.018$ ), suggesting that combination of datasets by meta-analysis may be overconservative for these data. On balance, however, we are cautious about concluding that there is evidence for non-additive interaction between SLC2A9 genotype and SSB consumption in gout risk, our data are an indication of non-additive interactions that require further supportive evidence in additional datasets. The ARIC serum urate analysis supported the gout data-a greater genotype-specific increase of serum urate was seen for C-allele carriers than non-carriers, with no differential effect seen with the consumption of artificially sweetened beverages (table 4). There was a trend towards significant evidence for interaction between SLC2A9 genotype and SSB (but not artificially sweetened beverage) consumption in the determination of serum urate levels (table 4; $\mathrm{p}_{\text {Interaction }}=0.062$ ). Collectively, these data suggest a nonadditive genotype-specific interaction between SSB consumption and SLC2A9 genotype, and imply a physiological mechanism whereby high simple sugar exposure derived from soft drinks over-rides the positive versus negative risk discrimination of the SLC2A9 gout risk alleles by interfering with the ability of SLC2A9 to transport uric acid.

It is worthwhile directly comparing our study to those of Choi et $a l^{8} 9$ who positively associated HFCS-sweetened beverage consumption with risk of incident gout, as the similarities and differences are illuminating. First, both the Choi et al studies were done in the North American population where the most common soft drink sweetener used is HFCS (HFCS-55), which is 55\% fructose and $42 \%$ glucose. In NZ the sweetener used is sucrose, which is equimolar fructose and glucose. Our data, therefore, broaden the generalisability of the role of non-artificially sweetened soft drink consumption, including to an ancestral group other than European Caucasian. Second, both Choi et al reports studied health professionals (the Health Professionals Study (HPS) in men $^{8}$ and the Nurses Health Study (NHS) in women ${ }^{9}$ ), 
Table 2 Risk of gout for sugar-sweetened beverage intake by ethnic group

\begin{tabular}{|c|c|c|c|c|c|c|c|c|c|}
\hline & \multirow{2}{*}{ Obs ( $\mathrm{n}$ controls, $\mathrm{n}$ cases) } & \multicolumn{8}{|c|}{ Frequency of SSB intake (servings/day) } \\
\hline & & 0 & 0.01 to $0.99^{*}$ & 1.0 to 1.99 & 2.0 to 2.99 & 3.0 to 3.99 & 4.0 to 4.99 & $5.0+$ & $P_{\text {Trend }}{ }^{t}$ \\
\hline \multicolumn{10}{|l|}{ NZ European Caucasian } \\
\hline Unadjusted OR (95\% Cl) & $592(180,412)$ & 1.00 & 1.53 (0.31 to 7.49$)$ & $0.79(0.52$ to 1.21$)$ & $1.51(0.78$ to 2.93$)$ & $0.65(0.30$ to 1.41$)$ & 3.93 (0.89 to 17.26$)$ & 1.05 (0.43 to 2.64$)$ & 0.56 \\
\hline$p$ Value & & - & 0.60 & 0.28 & 0.22 & 0.28 & 0.070 & 0.90 & \\
\hline Adjusted OR‡ (95\% Cl) & 563 & 1.00 & 2.17 (0.38 to 12.51$)$ & 1.11 (0.60 to 2.05$)$ & 2.82 (1.08 to 7.34$)$ & $1.42(0.47$ to 4.27$)$ & 6.89 (1.05 to 45.44$)$ & 2.38 (0.64 to 8.84$)$ & 0.020 \\
\hline $\mathrm{p}$ Value & & - & 0.39 & 0.75 & 0.034 & 0.53 & 0.045 & 0.20 & \\
\hline \multicolumn{10}{|l|}{ NZ Māori } \\
\hline Unadjusted OR (95\% Cl) & $502(312,190)$ & 1.00 & $1.14(0.35$ to 3.73$)$ & $0.66(0.40$ to 1.08$)$ & $0.69(0.36$ to 1.33$)$ & $1.22(0.63$ to 2.36$)$ & 1.94 (0.91 to 4.17$)$ & 1.34 (0.74 to 2.43$)$ & 0.17 \\
\hline$p$ Value & & - & 0.83 & 0.10 & 0.27 & 0.56 & 0.088 & 0.33 & \\
\hline Adjusted OR $¥(95 \% \mathrm{Cl})$ & 463 & 1.00 & $0.32(0.05$ to 1.91$)$ & $0.76(0.36$ to 1.58$)$ & $1.28(0.52$ to 3.14$)$ & $0.89(0.33$ to 2.35$)$ & $5.19(1.48$ to 18.17$)$ & $1.44(0.59$ to 3.53$)$ & 0.11 \\
\hline$p$ Value & & - & 0.21 & 0.46 & 0.59 & 0.81 & 0.010 & 0.42 & \\
\hline \multicolumn{10}{|l|}{ NZ Pacific Islander } \\
\hline Unadjusted OR (95\% Cl) & $536(217,319)$ & 1.00 & - & $0.85(0.50$ to 1.45$)$ & 0.84 (0.49 to 1.42$)$ & 0.84 (0.48 to 1.47$)$ & 2.42 (1.09 to 5.33$)$ & $1.38(0.76$ to 2.50$)$ & 0.16 \\
\hline $\mathrm{p}$ Value & & - & - & 0.55 & 0.51 & 0.53 & 0.029 & 0.29 & \\
\hline Adjusted OR $\ddagger(95 \% \mathrm{Cl})$ & 485 & 1.00 & - & $1.20(0.59$ to 2.46$)$ & $0.84(0.42$ to 1.69$)$ & $1.00(0.47$ to 2.13$)$ & 2.84 (1.04 to 7.77$)$ & 2.17 (0.98 to 4.77$)$ & 0.050 \\
\hline$p$ Value & & - & - & 0.62 & 0.63 & 1.00 & 0.043 & 0.055 & \\
\hline \multicolumn{10}{|l|}{ ARIC } \\
\hline Unadjusted OR (95\% Cl) & $7059(6911,148)$ & 1.00 & 1.71 (0.78 to 3.74$)$ & 1.54 (0.69 to 3.43 ) & $1.48(0.57$ to 3.83$)$ & $3.79(1.35$ to 10.61$)$ & - & $2.54(0.73$ to 8.80$)$ & 0.15 \\
\hline $\mathrm{p}$ Value & & - & 0.18 & 0.29 & 0.43 & 0.011 & - & 0.14 & \\
\hline Adjusted OR OR (95\% Cl) & 6705 & 1.00 & 1.50 (0.68 to 3.30$)$ & $1.36(0.61$ to 3.05$)$ & 1.19 (0.45 to 3.12$)$ & 3.05 (1.07 to 8.66$)$ & - & 2.31 (0.65 to 8.19$)$ & 0.26 \\
\hline $\mathrm{p}$ Value & & - & 0.31 & 0.46 & 0.73 & 0.037 & - & 0.20 & \\
\hline
\end{tabular}

*Selection of exposure groups is described in the Methods.

$t \mathrm{p}_{\text {Trend }}$ was the $\mathrm{p}$ value associated with the single line SSB variable in regression analysis.

(ARIC only). ARIC, Atherosclerosis Risk in Communities; NZ, New Zealand; SSB, sugar (sucrose)-sweetened beverage. 
Table 3 Sugar-sweetened beverage consumption and change in gout risk, with stratification by SLC2A9 genotype

\begin{tabular}{|c|c|c|c|c|c|c|}
\hline & & Obs & $\Delta$ In gout risk, $95 \% \mathrm{Cl}$ (odds ratio)* & $\mathbf{p}$ & $\boldsymbol{\beta}_{\text {Interaction }}(95 \% \mathrm{Cl}) \dagger$ & $\mathbf{p}$ \\
\hline \multirow[t]{3}{*}{ NZ European Caucasian } & Unstratified & 563 & $1.20(1.03$ to to 1.40$)$ & 0.020 & $0.021(-0.016$ to 0.058$)$ & 0.27 \\
\hline & $\mathrm{C}$ allele non-carrier & 373 & $1.16(0.95$ to 1.43$)$ & 0.14 & & \\
\hline & $\mathrm{C}$ allele carrier & 148 & $1.27(0.96$ to 1.67$)$ & 0.091 & & \\
\hline \multirow[t]{3}{*}{ NZ Māori } & Unstratified & 463 & $1.11(0.98$ to 1.27$)$ & 0.11 & $-0.064(-0.12$ to -0.011$)$ & 0.018 \\
\hline & C allele non-carrier & 396 & 1.19 (1.03 to 1.37$)$ & 0.019 & & \\
\hline & $\mathrm{C}$ allele carrier & 51 & $0.76(0.49$ to 1.16$)$ & 0.20 & & \\
\hline \multirow[t]{3}{*}{ NZ Pacific Islander } & Unstratified & 489 & $1.13(1.01$ to 1.27$)$ & 0.030 & $0.073(-0.018$ to 0.16$)$ & 0.12 \\
\hline & $\mathrm{C}$ allele non-carrier & 454 & $1.11(0.99$ to 1.25$)$ & 0.039 & & \\
\hline & $\mathrm{C}$ allele carrier & 27 & $1.71(0.85$ to 3.42$)$ & 0.13 & & \\
\hline \multirow[t]{3}{*}{ ARIC } & Unstratified & 6721 & 1.09 (0.94 to 1.27$)$ & 0.26 & $0.00028(-0.0067$ to 0.0072$)$ & 0.94 \\
\hline & $\mathrm{G}$ allele non-carrierł & 4082 & 1.05 (0.88 to 1.26$)$ & 0.58 & & \\
\hline & G allele carrier & 2639 & $1.16(0.88$ to 1.54$)$ & 0.29 & & \\
\hline \multirow[t]{3}{*}{ All Populations Combined } & Unstratified & 8236 & $1.13(1.06$ to 1.20$)$ & $1.9 \times 10^{-4}$ & $-0.013^{\S}(-0.022$ to -0.003$)$ & 0.010 \\
\hline & Minor allele non-carrierł & 5305 & $1.12(1.05$ to 1.21$)$ & 0.001 & & \\
\hline & $\mathrm{C}$ allele carrier & 2888 & 1.15 (0.98 to 1.34$)$ & 0.082 & & \\
\hline
\end{tabular}

whereas our study was not targeted to any particular demographic. One statistic that reflects this demographic difference is the prevalence of higher SSB consumption in the NZ datasets. In the HPS, $2.3 \%$ of participants consumed $\geq 2$ drinks/day, and in the NHS, $1.9 \%$ consumed $\geq 2$ /day. In our sample set, $18.9 \%$ of European Caucasian controls, $34.4 \%$ of Māori controls and $56.1 \%$ of Pacific Island controls consumed $\geq 2$ /day. Third, our study was on prevalent gout, not incident gout in the case of the studies of Choi et $a l^{89}$ (although some incident cases may have been included in the ARIC samples (see online supplemental material)). From the incident gout studies it is possible to conclude that HFCS-sweetened beverage consumption was causative of gout, and should be targeted in primary prevention. By contrast, given our study was of cross-sectional design on prevalent gout, our results are more consistent with a contribution to established gout by SSB consumption. Collectively, this evidence strongly supports recommending reduction in SSB in primary prevention of gout, ${ }^{8} 9$ and also in management of patients with established gout, as an adjunct to urate-lowering therapy.
One weakness in our study is the incompleteness of our dietary data, meaning we cannot address whether or not consumption of larger quantities of SSB is a marker for other dietary risk factors for hyperuricaemia and gout - a diet high in purine-rich foods, alcohol and vitamin $\mathrm{A}$, and low in vitamin $\mathrm{C}$ and coffee. ${ }^{18-22}$ Given our data were adjusted for alcohol consumption, we are confident that this risk factor is not a confounder in the SSB-gout association. The studies reporting association between HFCS-sweetened soft drink consumption and serum urate levels and gout in men took into account consumption of meat, seafood, purine-rich vegetables and total vitamin $C,{ }^{8}{ }^{10}$ suggesting that serum urate levels and gout risk are independently influenced by soft drink consumption. Although not a weakness in our study per se, rather a weakness inherent in case-control studies in general, it is possible that SSB consumption is a marker for a separate causal environmental risk factor. Arguing against this possibility is the biological plausibility of SSB consumption as a causal factor in gout, and some evidence for interaction with the simple sugar and uric acid transporter, SLC2A9.

Table 4 Sugar-sweetened and artificially sweetened beverage consumption and change in serum urate levels in ARIC controls, with stratification by SLC2A9 genotype

\begin{tabular}{|c|c|c|c|c|c|c|}
\hline Sweetener & & Obs & $\Delta$ In serum urate, $95 \% \mathrm{Cl}(\mathrm{mmol} / \mathrm{L})^{*}$ & $\mathrm{p}$ Value & $\boldsymbol{\beta}_{\text {Interaction, }}$ 95\% Cl${ }^{\dagger}$ & $\mathrm{p}$ Value \\
\hline \multirow[t]{3}{*}{ HFCS } & Unstratified & 6574 & $0.003(0.002$ to 0.005$)$ & $6.9 \times 10^{-6}$ & $0.003(-0.000$ to 0.059$)$ & 0.062 \\
\hline & C allele non-carrier $\ddagger$ & 3977 & 0.002 (0.000 to 0.004$)$ & 0.016 & & \\
\hline & $\mathrm{C}$ allele carrier & 2597 & 0.005 (0.003 to 0.007$)$ & $8.7 \times 10^{-5}$ & & \\
\hline \multirow[t]{3}{*}{ Artificial } & Unstratified & 6574 & $0.000(-0.000$ to 0.001$)$ & 0.48 & $-0.001(-0.002$ to 0.001$)$ & 0.39 \\
\hline & C allele non-carrier $\ddagger$ & 3977 & $0.000(-0.000$ to 0.001$)$ & 0.41 & & \\
\hline & $\mathrm{C}$ allele carrier & 2597 & $-0.004(-0.013$ to 0.006$)$ & 0.45 & & \\
\hline
\end{tabular}

*Change per increase in consumption category (as defined for the NZ data) adjusted by age, sex, BMI, alcohol (continuous variable), fruit intake (continuous variable), kidney disease, high blood pressure and relatedness.

tBeverage consumption per day ordinal (ordered) variables $(0,1,2,3,4,5,6)$ were created as described in the Supplementary Material and used as a continuous variable to derive the consumption by genotype interaction term with serum urate as outcome.

¥Genotyped with rs11942223 surrogate $r$ s 6449173 (major allele A, minor allele C) - $r^{2}=1$ with $r s 11942223$ in European Caucasian.

ARIC, Atherosclerosis Risk in Communities; NZ, New Zealand; HFCS, high fructose corn syrup; Obs, Observations. 
SLC2A9 is expressed as two isoforms ${ }^{23}$-full-length (SLC2A9v1) and a variant missing 28 N-terminal residues (SLC2A9v2). SLC2A9v1 is expressed on the basolateral (blood) membrane, and SLC2A9v2 is expressed on the apical (reabsorption) membrane of the renal proximal tubule. ${ }^{24}$ There is a positive correlation between serum urate levels and SLC2A9v2 expression, but not for SLC2A9v1. ${ }^{25}$ If genotype at $r s 11942223$ correlates with expression of the different isoforms, ${ }^{26}$ then the interaction data can be viewed in a different context, as differential reaction of two structurally distinct proteins, albeit encoded by the same gene, to increased SSB consumption; one isoform (SLC2A9v2, encoded by the major serum urate-raising allele) reacts in a simple additive fashion and the other (SLC2A9v1, encoded by the minor serum urate-lowering allele) in a not straightforward non-additive way. From the current state of knowledge and given the complexity of urate transport in the renal tubule it is difficult to propose a plausible mechanism to explain any non-additive interaction (under the assumption that this risk is mediated through a change in serum urate level). It is known that individuals of European Caucasian ancestry positive for the gout-protective C-allele at $r s 11942223$ exhibit a reduced change in serum urate and increased FEUA in response to an acute fructose load, with no genotype-specific effect in people of NZ Māori and Pacific Islander (Polynesian) ancestry. ${ }^{16}$ These data are inconsistent with the observation of increased serum urate and risk of gout in C-allele carrying individuals of European Caucasian and Polynesian ancestry upon exposure to fructose-containing SSB (tables 3 and 4), suggesting that distinct biological mechanisms underlie the ref 16 observation and the interaction data reported herein. Chronic exposure to fructosecontaining SSB would more likely involve other mechanisms (eg, epigenetic) that influence the expression and activity of SLC2A9.

In summary, our data support the recommendation that reduction of SSB consumption should be pursued in established gout. The collective data suggest a non-additive genotypespecific interaction between SSB consumption and SLC2A9 genotype and imply a physiological mechanism whereby high simple sugar exposure derived from soft drinks interferes with the ability of SLC2A9 to transport uric acid.

\begin{abstract}
Acknowledgements This work was supported by the Health Research Council of New Zealand, Arthritis New Zealand, New Zealand Lottery Health and the University of Otago. No funder had direct input into the design and conduct of the study; collection, management, analysis, and interpretation of the data; and preparation, review, or approval of the manuscript. The Atherosclerosis Risk in Communities study analysis (project \#834) was approved by the relevant Database of Genotype and Phenotype (dbGaP; http://www.ncbi.nim.nih/gov/dbgap) Data Access Committee. The Atherosclerosis Risk in Communities Study is carried out as a collaborative study supported by National Heart, Lung, and Blood Institute contracts N01-HC-55015, N01-HC-55016, N01-HC-55018, N01-HC-55019, N01-HC-55020, N01-HC-55021, N01-HC-55022, R01HL087641, R01HL59367 and R01HL086694; National Human Genome Research Institute contract U01HG004402; and National Institutes of Health contract HHSN268200625226C. The authors thank the staff and participants of the ARIC study for their important contributions. Infrastructure was partly supported by Grant Number UL1RR025005, a component of the National Institutes of Health and NIH Roadmap for Medical Research. TRM had full access to all of the data in the study and takes responsibility for the integrity of the data and the accuracy of the data analysis. All authors declare no conflicts of interest, including relevant financial interests, activities, relationships, and affiliations relevant to the subject of this manuscript.
\end{abstract}

Contributors All authors provided a significant contribution either to conception and design, acquisition of data and/or analysis and interpretation of data. All authors provided a significant contribution to the drafting of the manuscript and to intellectual content. All authors approved the final manuscript.

Funding New Zealand Health Research Council grant numbers 08/075 and 11/ 1075.

\section{Competing interests None.}

\section{Patient consent Obtained.}

Ethics approval The New Zealand MultiRegion Ethics Committee.

Provenance and peer review Not commissioned; externally peer reviewed.

Open Access This is an Open Access article distributed in accordance with the Creative Commons Attribution Non Commercial (CC BY-NC 3.0) license, which permits others to distribute, remix, adapt, build upon this work non-commercially, and license their derivative works on different terms, provided the original work is properly cited and the use is non-commercial. See: http://creativecommons.org/ licenses/by-nc/3.0/

\section{REFERENCES}

1 Campion EW, Glynn RJ, DeLabry LO. Asymptomatic hyperuricemia. Risks and consequences in the Normative Aging Study. Am J Med 1987;82:421-6.

2 Sorensen LB, Levinson DJ. Origin and extrarenal elimination of uric acid in man. Nephron 1975;14:7-20.

3 Merriman TR, Dalbeth $\mathrm{N}$. The genetic basis of hyperuricaemia and gout. Joint Bone Spine 2011;78:35-40.

4 Köttgen A, Albrecht E, Teumer A, et al. Genome-wide association analyses identify 18 new loci associated with serum urate concentrations. Nat Genet 2013;45:145-54

5 Caulfield MJ, Munroe PB, O'Neill D, et al. SLC2A9 is a high-capacity urate transporter in humans. PLOS Med 2008; 5:e197.

6 Witkowska K, Smith KM, Yao SY, et al. Human SLC2A9a and SLC2A9b isoforms mediate electrogenic transport of urate with different characteristics in the presence of hexoses. Am J Physiol Renal Physiol 2012;303:F527-39.

7 Hollis-Moffatt JE, Xu X, Dalbeth N, et al. A role for the urate transporter SLC2A9 gene in susceptibility to gout in New Zealand Māori, Pacific Island and Caucasian case-control cohorts. Arthritis Rheum 2009;60:3485-92.

8 Choi HK, Curhan G. Soft drinks, fructose consumption, and the risk of gout in men: prospective cohort study. Br Med J 2008;336:309-12.

9 Choi HK, Willett W, Curhan G. Fructose-rich beverages and risk of gout in women. JAMA 2010;304:2270-8.

10 Choi JW, Ford ES, Gao X, et al. Sugar-sweetened soft drinks, diet soft drinks, and serum uric acid level: the Third National Health and Nutrition Examination Survey. Arthritis Rheum 2008;59:109-16.

11 Perheentupa J, Raivio K. Fructose-induced hyperuricaemia. Lancet 1967;2: 528-31.

12 Wang DD, Sievenpiper JL, de Souza RJ, et al. The effects of fructose intake on serum uric acid vary among controlled dietary trials. J Nutr 2012;142: 916-23.

13 Lanaspa MA, Tapia E, Soto V, et al. Uric acid and fructose: potential biological mechanisms. Semin Nephrol 2011;31:426-32.

14 Johnson RJ, Perez-Pozo SE, Sautin YY, et al. Hypothesis: could excessive fructose intake and uric acid cause type 2 diabetes? Endocr Rev 2009;30:96-116.

15 Le MT, Frye RF, Rivard CJ, et al. Effects of high-fructose corn syrup and sucrose on the pharmacokinetics of fructose and acute metabolic and hemodynamic responses in healthy subjects. Metabolism 2012;61:641-51.

16 Dalbeth N, House ME, Gamble GD, et al. Population-specific influence of SLC2A9 genotype 0 the acute hyperuricemia response to a fructose load. Ann Rheum Dis 2013;72:1868-73.

17 Wallace SL, Robinson H, Masi AT, et al. Preliminary criteria for the classification of the acute arthritis of primary gout. Arthritis Rheum 1977;20:895-900.

18 Choi HK, Atkinson K, Karlson EW, et al. Purine-rich foods, dairy and protein intake, and the risk of gout in men. N Engl J Med 2004;350:1093-103.

19 Choi HK, Atkinson K, Karlson EW, et al. Alcohol intake and risk of incident gout in men: a prospective study. Lancet 2004;363:1277-81.

20 Choi HK, Gao X, Curhan G. Vitamin C intake and the risk of gout in men: a prospective study. Arch Intern Med 2009;169:502-7.

21 Choi WJ, Ford ES, Curhan G, et al. Independent association of serum retinol and $\beta$-carotene levels with hyperuricemia: a national population study. Arthritis Care Res 2012:64:389-96.

22 Choi HK, Willett W, Curhan G. Coffee consumption and risk of incident gout in men: a prospective study. Arthritis Rheum 2007;56:2049-55.

23 Augustin R, Carayannopoulos MO, Dowd LO, et al. Identification and characterization of human glucose transporter-like protein-9 (GLUT9): alternative splicing alters trafficking. J Biol Chem 2004;279:16229-36.

24 Bibert S, Hess SK, Firsov D, et al. Mouse GLUT9: evidences for a urate uniporter. Am J Physiol Renal Physiol 2009;297:F612-19.

25 Döring A, Gieger C, Mehta D, et al. SLC2A9 influences uric acid concentrations with pronounced sex-specific effects. Nat Genet 2008;40:430-6.

26 Merriman TR. Population heterogeneity in the genetic control of serum urate. Semin Nephrol. 2011;31:420-5 